\title{
Glucose imaging is not just $\left[{ }^{18} \mathrm{~F}\right] \mathrm{FDG}$ !
}

\author{
SRSTT Radiopharmaconnect ${ }^{1}$ \\ ${ }^{1}$ Radiopharmaconnect
}

August 6, 2020

by Benjamin Rotstein

Imaging glucose utilization is a mainstay and the most well-known application of positron emission tomography (PET) imaging. If one had to choose a single tracer that headlines the technology, surely many would select $\left[{ }^{18} \mathrm{~F}\right] 2$-deoxy-2-fluoro-d-glucose $\left(\left[{ }^{18} \mathrm{~F}\right] \mathrm{FDG}\right)$.

But imaging with ${ }^{18}$ FJFDG does not reflect the total picture of glucose distribution and metabolism in vivo; it mostly tells us about the facilitated glucose transporters GLUTs, and especially GLUT1, which provides basal glucose supply to almost all tissues.

Over the last several years, a group out of University of California Los Angeles (UCLA) has been developing radiotracers for the sodium-glucose linked transporters (SGLTs). Methyl $\left[{ }^{18} \mathrm{~F}\right] 4$-deoxy-4-fluoro- $\alpha$-Dglucosepyranoside $\left(\left[{ }^{18} \mathrm{~F}\right] \mathrm{Me}-4 \mathrm{FDG}\right)$ is also a glucose derivative, but is a poor substrate for the GLUTs and has greater affinity for SGLT1 and SGLT2. Similar to $\left[{ }^{18} \mathrm{~F}\right] \mathrm{FDG},\left[{ }^{18} \mathrm{~F}\right] \mathrm{Me}-4 \mathrm{FDG}$ is effectively trapped intracellularly as it is not a substrate for further downstream metabolism and due to low rate of exit through SGLTs.
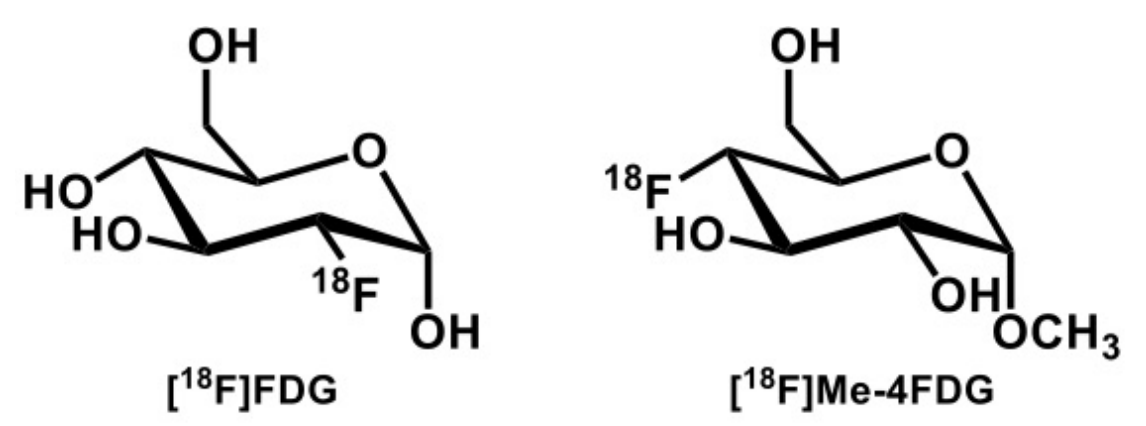

Figure 1: Structures of $\left[{ }^{18} \mathrm{~F}\right] \mathrm{FDG}$ and $\left[{ }^{18} \mathrm{~F}\right] \mathrm{Me}-4 \mathrm{FDG}$

PET imaging with $\left[{ }^{18} \mathrm{~F}\right] \mathrm{Me}-4 \mathrm{FDG}$ has provided some interesting complementarity to $\left[{ }^{18} \mathrm{~F}\right] \mathrm{FDG}$. $\left[{ }^{18} \mathrm{~F}\right] \mathrm{Me}-$ 4 FDG shows comparatively low uptake into the kidneys and urinary excretion compared to $\left[{ }^{18} \mathrm{~F}\right] \mathrm{FDG}$ due to reabsorption from the glomerular filtrate by SGLTs. Since the blood-brain barrier uses primarily GLUT1 and not SGLTs, $\left[{ }^{18} \mathrm{~F}\right] \mathrm{Me}-4 \mathrm{FDG}$ shows only low brain uptake under normal conditions. This offers an opportunity in cases such as astrocytoma, where $\left[{ }^{18} \mathrm{~F}\right] \mathrm{FDG}$ struggles to achieve contrast due to high baseline brain uptake of the tracer. Importantly, the researchers found evidence to support SGLT2 expression in the proliferating 
tumor microvasculature, suggesting a mechanism for selective uptake. This work was published last year in Journal of Neuro-Oncology (DOI: 10.1007/s11060-018-2823-7).

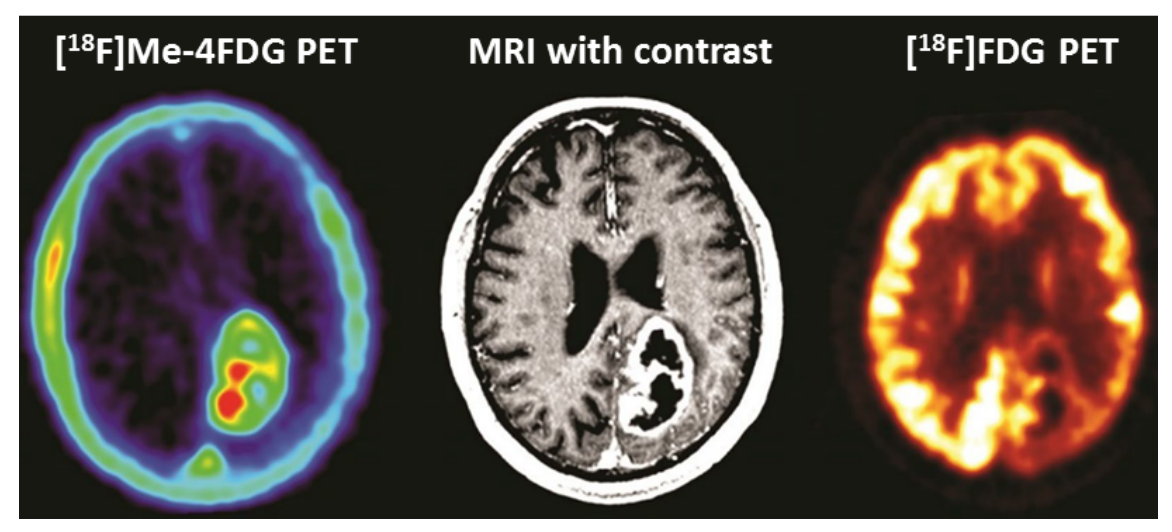

Figure 2: $\left[{ }^{18} \mathrm{~F}\right] \mathrm{Me}-4 \mathrm{FDG}$ PET, gadolinium contrast MRI, and $\left[{ }^{18} \mathrm{~F}\right] \mathrm{FDG}$ PET images from a WHO Grade IV glioblastoma patient. Reproduced from Journal of Neuro-Oncology 2018, 138, 557-569.

In a second application, $\left[{ }^{18} \mathrm{~F}\right] \mathrm{Me}-4 \mathrm{FDG}$ was used to identify early-stage lung adenocarcinoma (LADC) using PET. $\left[{ }^{18} \mathrm{~F}\right] \mathrm{FDG}$ struggles to identify early invasive disease because of relatively low GLUT expression and glucose utilization. This is consistent with increased GLUT expression correlating to tumor progression towards poorly differentiated "high-grade" LADC. In contrast, cells in early stage "low-grade" lesions primarily use SGLT2 for glucose transport. With this in mind, $\left[{ }^{18} \mathrm{~F}\right] \mathrm{Me}-4 \mathrm{FDG}$ was able to detect lung tumors at an earlier stage than $\left[{ }^{18} \mathrm{~F}\right] \mathrm{FDG}$ in genetically engineered mouse models, with sustained uptake along disease progression. Check this out and their work with patient-derived xenografts at Science Translational Medicine (DOI: 10.1126/scitranslmed.aat5933).

In addition to the potential for earlier and more sensitive detection of tumors, $\left[{ }^{18} \mathrm{~F}\right] \mathrm{Me}-4 \mathrm{FDG}$ also points to many new potential lines of inquiry. For example, recently several SGLT2 inhibitors have reached the market as treatments for type II diabetes mellitus. Based on the above results, this class of drugs (gliflozins) may also hold potential for treatment of certain types of cancer at a stage when they are dependent on this "alternative" glucose transporter. Indeed, the Science Translational Medicine paper discussed above contains a preclinical trial towards that end.

For myself, the $\left[{ }^{18} \mathrm{~F}\right] \mathrm{Me}-4 \mathrm{FDG}$ story so far is a nice reminder that in molecular imaging it is not just the functional outcomes but also the mechanisms that matter.

Benjamin Rotstein is an Assistant Professor at University of Ottawa and Scientist at University of Ottawa Heart Institute. His lab conducts research into synthetic radiochemical methods with PET isotopes as well as radiotracer development, with an emphasis on cardiovascular applications. Follow their progress at rotsteinlab.com 